\title{
A Calcified Retroperitoneal Teratoma in a Postmenopausal Woman
}

\author{
Abu Atileh Luay, MD,1,2 Hnin Thazin Htut, MSC (Ob/Gy), 2,3 and Kuan-Gen Huang, MD²,4
}

\begin{abstract}
Background: This article describes a very rare entity-a mature cystic teratoma occurring in the retroperitoneum in a postmenopausal patient, which is also a rare age for this presentation.

Case: A 51-year-old postmenopausal woman presented with a history of low-back pain that radiated to a lower limb. During her evaluation, a mature cystic teratoma was found in the retroperitoneal region with calcification. She was operated on to remove adhesions that she had and to remove the teratoma.

Results: This patient's low-back pain was resolved after her operation.

Conclusions: Although rare, a retroperitoneal teratoma can present in postmenopause, usually in a patient with nonspecific complaints. At this age of presentation, malignancy should always be ruled out by surgical excision and careful histologic examination of the excised specimen. (J GYNECOL SURG 37:60)
\end{abstract}

Keywords: teratoma, dermoid cyst, retroperitoneal teratoma, postmenopausal teratoma

\section{Introduction}

$\mathbf{M}$ ATURE CYSTIC TERATOMAS are rare germ-cell tumors of the ovary, comprised of all germ-cell layersectoderm, mesoderm, and endoderm. ${ }^{1}$ These tumors account for $\sim 25 \%$ of all ovarian tumors but not more than $5 \%$ of malignant ovarian tumors. Mature cystic teratomas often occur in younger patients, with an average age of $30 .{ }^{1}$ These tumors are typically evident within the pelvis because of their fat and calcific density. ${ }^{1,2}$ The presenting case is a rare retroperitoneal teratoma in an older woman. These tumors are uncommon in postmenopausal patients. In addition, this case also demonstrates the detection of the tumor and the proper referral of a female patient with a calcific mass.

\section{Case}

A 51-year-old, para 4, postmenopausal female (menopause attained 9 years back) was referred from an orthopedic department for a 2-month history of low-back pain, after exclusion of musculoskeletal and neurologic causes and a finding of a right pelvic mass with bone formation noted on a KUB [kidneys, ureters, and bladder] X-ray (Fig. 1).
This patient complained of a dull, aching lower-back pain with right lower-limb numbness that was not associated with abdominal pain, change in bowel habits, urinary symptoms, nor vaginal discharge.

Her general physical examination was unremarkable, and her body mass index was 24. Systemic and neurologic examinations yielded normal findings. On abdominal examination, this patient's abdomen was soft with no guarding nor rigidity. There was a nontender, smooth-surfaced, firm lump, $10 \times 8 \mathrm{~cm}$, with restricted mobility that was palpated in the right iliac fossa. Pelvic examination showed a normal-size uterus with a mass in the anterior and right fornixes.

Pelvic ultrasound showed a right ovarian mass of $10.5 \mathrm{~cm}$ in diameter with echogenic shadowing that indicated a calcified component. All laboratory investigations, including tumor markers, such as cancer antigen (CA)-125, $\alpha$-fetoprotein, and CA $19-9$ were $30.2 \mathrm{U} / \mathrm{mL}, 4 \mathrm{ng} / \mathrm{mL}$, and $25 \mathrm{U} / \mathrm{mL}$, respectively-within normal ranges.

With a preoperative diagnosis of an ovarian tumor in this postmenopausal woman, she was counseled about a total laparoscopic hysterectomy (TLH) with a bilateral salpingooophorectomy (BSO).

A 4-handed surgery was started through the Lee-Huang point. Intraoperative findings showed a normal-size uterus, a

\footnotetext{
${ }^{1}$ Department of Obstetrics and Gynecology, Albasheer Hospital, Amman, Jordan.

${ }^{2}$ Department of Obstetrics and Gynecology, Chang Gung Memorial Hospital at Linkou, Kweishan, Taoyuan, Taiwan.

${ }^{3}$ Department of Obstetrics and Gynecology, Faculty of Medicine, University of Medicine, Yangon, Myanmar.

${ }^{4}$ Department of Obstetrics and Gynecology, Chang Gung University College of Medicine, Kweishan, Taoyuan, Taiwan.
} 


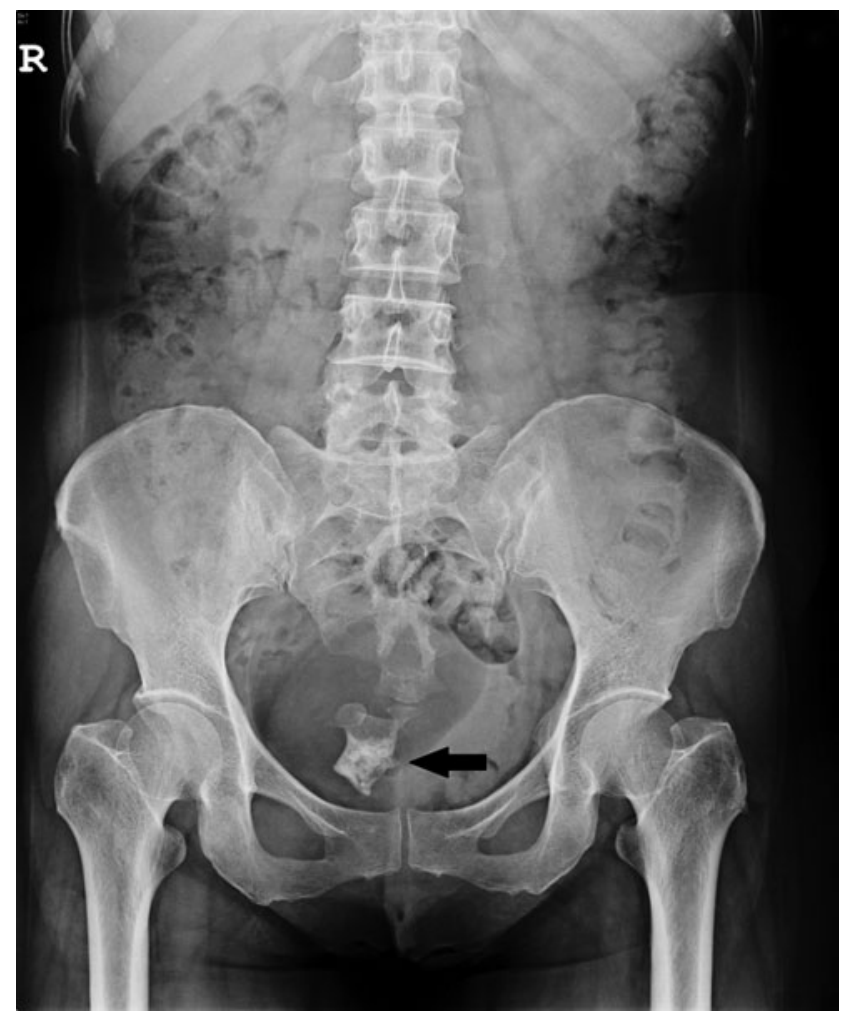

FIG. 1. KUB [kidneys, ureters, and bladder] X-ray film where the arrow points to the irregular calcification pattern of a retroperitoneal teratoma.

normal left ovary, and a right retroperitoneal mass of $\sim 10 \mathrm{~cm}$ in diameter reaching the presacral region. The mass was densely adhered to the rectum and right ureter. First, adhesiolysis was done to identify the rectum and right ureter. Then, the retroperitoneal mass was excised using LigaSure ${ }^{\text {tm }}$ and a monopolar scissor to cut the bony elements and calcified tissue. After that, a TLH and a left salpingo-oophorectomy were performed. During the operation, bleeding in the presacral region was controlled with hemoclips (Fig. 2).

The gross specimen was comprised of 2 pieces of tissue measuring $8.3 \times 5.7 \times 5.5 \mathrm{~cm}$ and $6.3 \times 1.9 \times 1.4 \mathrm{~cm}$ (Fig. 3). There was hair, fatty tissue, bony tissue, and sebaceous content inside the mass. A histopathology examination revealed a cystic tumor comprised of squamous epithelium, respiratorytype ciliated columnar epithelium, skin appendages, mature fat tissue, lamellar bone with bone-marrow spaces, and some melanin pigments-all indicating that the mass was a mature cystic teratoma.

\section{Results}

This patient's low back pain was relieved after her operation.

\section{Discussion}

Teratomas are germ-cell tumors that develop from the 3 embryonic germ cell layers. ${ }^{1}$ Grossly, teratomas might be cystic or solid. Cystic teratomas are composed of completely mature parts and include sebaceous material and hair. These tumors are mostly benign. Solid teratomas, in

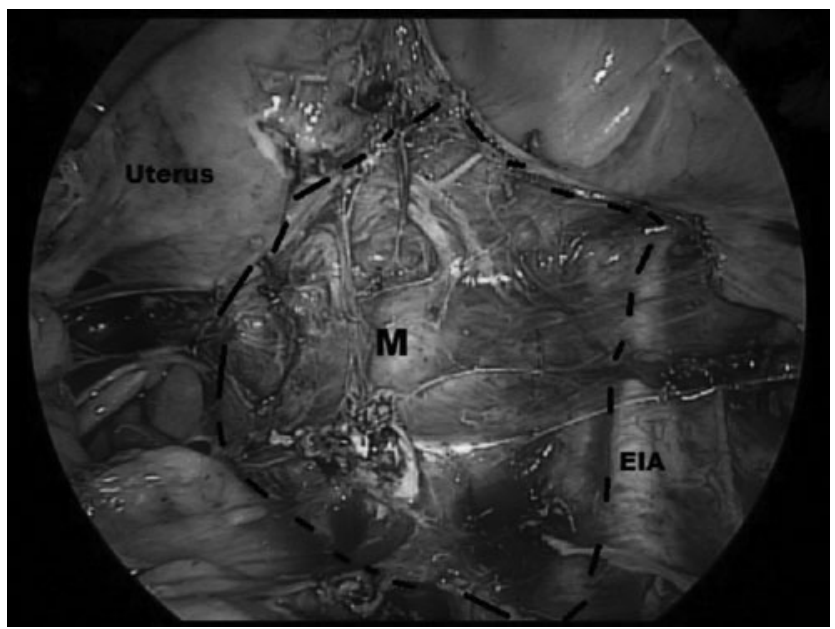

FIG. 2. Laparoscopic view of the pelvis showing the uterus, $M$ represents the retroperitoneal teratoma with its border demarcated by black short lines, reaching the right external iliac artery (EIA), with obliteration of the cul de sac.

most cases, are malignant and comprised of immature embryonic tissue. ${ }^{3}$ Many researchers reported that $1 \%$ of mature cystic teratomas undergo malignant transformation, mostly into squamous-cell carcinoma. ${ }^{4}$

Extragonadal teratomas are suggested to arise from primordial germ cells or early embryonic cells ${ }^{5,6}$; some of these tumors are primary retroperitoneal teratomas. These teratomas represent $\sim 1 \%-11 \%$ of all retroperitoneal tumors. They appear most commonly in neonates and young adults ${ }^{7}$. These teratomas have a bimodal age peak, as they tend to occur in the first 6 months of life and in early adulthood. Approximately $43 \%-55 \%$ of retroperitoneal teratomas are diagnosed within the first year of life, and less than 10\%-20\% present in patients older than $30 .^{8}$

Benign teratomas are usually diagnosed as incidental findings, unless these teratomas grow large, causing obstructive symptoms to develop. However, common presenting symptoms include back pain or abdominal pain.9

Many radiologic findings can indicate the diagnosis of teratoma, such as a complex lesion containing a well-circumscribed fluid component, fatty regions, and calcifications. ${ }^{10}$ In more

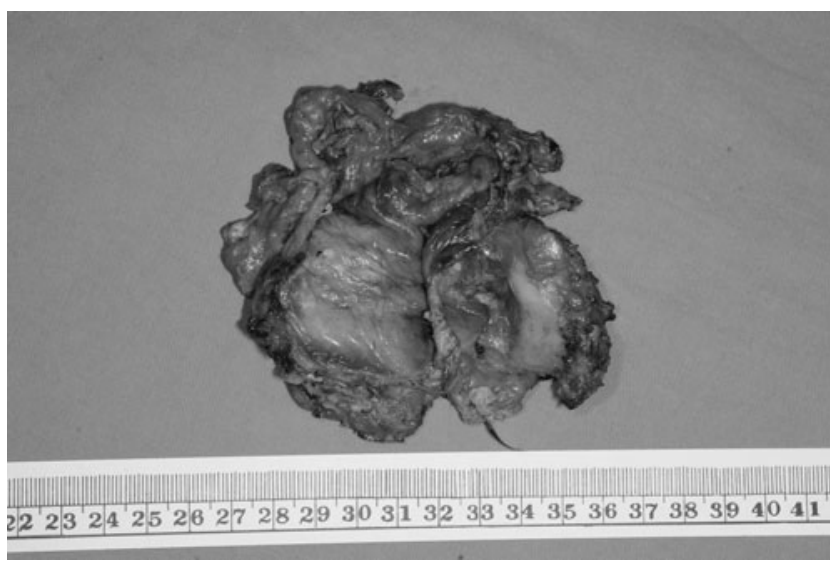

FIG 3. The gross specimen showed that the calcified retroperitoneal teratoma was $\sim 9 \mathrm{~cm}$. 
than half of these cases, plain X-ray films can show irregular calcifications, representing a calcific rim of the cyst wall or even a calcific structure, such as a tooth or a bony element. ${ }^{11}$ Approximately $12.5 \%$ of calcified tumors are malignant. ${ }^{3}$ However, a definitive diagnosis can only be made after surgical resection and careful histologic evaluation of the excised mass. ${ }^{10}$ As the current patient was postmenopausal, a TLH and a BSO were performed.

\section{Conclusions}

A retroperitoneal teratoma is a rare development. Although most commonly encountered in infants, children, and young adults, it can also present in a postmenopausal woman with nonspecific complaints. Malignancy should always be ruled out by surgical excision and careful histopathologic examination of the excised specimen.

\section{Acknowledgments}

The authors thank the Asia-Pacific Association for Gynecologic Endoscopy and Minimally Invasive Therapy for providing the International Fellowship Endoscopy Training Program at Chang Gung Memorial Hospital at Linkou, Kweishan, Taoyuan, Taiwan.

\section{Funding Information}

No funding was received from any agency or institution.

\section{References}

1. Gatcome HG, Assikis V, Kooby D, Johnstone PA. Primary retroperitoneal teratomas: A review of the literature. J Surg Oncol 2004;86:107.

2. Bellin MF, Duron JJ, Curet P, et al. Primary retroperitoneal teratoma in the adult: Correlation of MRI features with CT and pathology. Magn Reason Imaging 1991;9:263.
3. Bruneton JN, Diard F, Drouillard JP, et al. Primary retroperitoneal teratoma in adults: Presentation of two cases and review of the literature. Radiology 1980;134:613.

4. Renato F, Paolo V, Girolamo M, et al. Malignant retroperitoneal teratoma: Case report and literature review. Acta Urol Belg 1996;64:49.

5. Saiga T, Osasa H, Hatayama H, et al. The origin of extragonadal teratoma: Case report of an immature teratoma occurring in a prenatal brain. Pediatr Pathol 1991; $11: 759$.

6. Wagner H, Baretton GB, Schneiderbanger K, et al. Sex chromosome determination in extragonadal teratomas by interphase cytogenetics: Clues to histogenesis. Pediatr Pathol Lab Med 1997;17:401.

7. Wang RM, Chen CA. Primary retroperitoneal teratoma. Acta Obstet Gynecol Scand 2000;79:707.

8. Panageas E. General diagnosis case of the day: Primary retroperitoneal teratoma. Am J Roentgenol 1991;156:1292.

9. Lambrianides AL, Walker MM, Rosin RD. Primary retroperitoneal teratoma in adults. Urology 1987;29:310.

10. Davidson AJ, Hartman DS, Goldman SM. Mature teratoma of the retroperitoneum: Radiologic, pathologic, and clinical correlation. Radiology 1989;172:421.

11. Gschwend J, Burke TW, Woodward JE, et al. Retroperitoneal teratoma presenting as an abdominal-pelvic mass. Obstet Gynecol 1987;70:500.

Address correspondence to: Kuan-Gen Huang, MD

Department of Obstetrics and Gynecology Chang Gung Memorial Hospital at Linkou Medical Center and Chang Gung University College of Medicine 5 Fu-Hsin Street Kweishan, Taoyuan 333 Taiwan

E-mail:kghuang@ms57.hinet.net 